\title{
Nouvelle approche du comblement sinusien : analyse clinique et histologique
}

\author{
Younes $\mathrm{R}^{1}$, Nader $\mathrm{N}^{2}$ \\ ${ }^{1}$ Service de Chirurgie orale, Université Saint Joseph, Beyrouth, Liban \\ ${ }^{2}$ Service de Chirugie orale et maxillo-faciale, Université libanaise, Beyrouth, Liban
}

Introduction : Plusieurs substituts osseux d'origines diverses ont été utilisés dans le sinus pour traiter les déficiences osseuses du maxillaire postérieur. Une précédente étude (Froum et al. 2006) utilisant un os humain déprotéiné sous forme de particules spongieuses (Puros cancellous $^{\circledR}$, Tutogen Medical $\mathrm{GmbH}$ ) a donné des résultats prometteurs pour la néoformation osseuse. Ce substitut osseux est obtenu par un nouveau procédé de préparation (Tutoplast ${ }^{\circledR}$ ) réalisé en cinq étapes, permettant de préserver la structure collagénique de l'os. Utilisé depuis plus de trente ans en orthopédie, il se présente sous deux formes, corticale et spongieuse. Le but de la présente étude est d'évaluer, à 5 et 8 mois, le pourcentage d'os vital néoformé après une greffe sinusienne utilisant des particules allogéniques sous forme corticale.

Méthodes : 13 patients nécessitant 15 greffes sinusiennes par voie externe (Tatum 1977, Boyne et James 1980) ont été inclus dans une étude prospective utilisant des particules corticales d'os humain déprotéiné (Puros cortical ${ }^{\circledR}$, Tutogen Medical GmbH). A la $23^{\text {ème }}$ semaine post-greffe, simultanément à la pose d'implants enfouis, des carottes osseuses ont été prélevées à l'aide de tréphines dans les 15 sinus greffés. A la $36^{\text {ème }}$ semaine post-greffe, de nouvelles carottes osseuses ont été prélevées au cours du deuxième temps chirurgical. Toutes les biopsies osseuses ont subi après leur inclusion (paraffine et résine méthacrylate) des colorations spéciales en fonction de la technique d'inclusion utilisée.

Résultats : L'analyse histologique et histomorphométrique, utilisant à la fois les techniques dites décalcifiées et non décalcifiées, a montré, après 23 et 36 semaines, un pourcentage moyen respectivement de $14.32 \%$ et de $32.51 \%$ pour l'os vital et de $17.13 \%$ et de $7.65 \%$ pour les particules résiduelles.

Conclusions : Après 36 semaines, le substitut osseux allogénique sous forme corticale était entouré par un tissu osseux néoformé avec un faible pourcentage de particules résiduelles. Les résultats de cette étude montrent que l'os humain déprotéiné sous sa forme corticale peut être utilisé de façon satisfaisante pour les greffes sinusiennes.

Correspondance : ronald.younes@hotmail.com 